\title{
Carbon Nanotube Chirality Enrichment through Chirality-Selective Precipitation
}

\author{
Antonio Setaro ${ }^{*, 1}$, Pascal Bluemmel ${ }^{1}$, Marcus Witt ${ }^{1}$, Rohit Narula ${ }^{1,2}$, and Stephanie Reich \\ ${ }^{1}$ Department of Physics, Freie Universität Berlin, Arnimalee 14, 14195 Berlin, Germany. \\ ${ }^{2}$ Department of Physics, Indian Institute of Technology Delhi, Hauz Khas, New Delhi 110016, India.
}

Keywords SWNTs, selective enrichment, colloidal systems.

The interaction with surfactants changes from one carbon nanotube species to the other. We used this to replace nonionic surfactants by ionic ones. By fine-tuning the replacement conditions, we covered some specific nanotubes with non-ionic surfactants and the others with ionic ones. Addition of salt triggers precipitation of the species suspended by the ionic surfactant; this effectively leads to chiral selective enrichment of a carbon nanotube suspension. We hence produce an $(8,4)$-enriched suspension by replacing a polyglycerol-based custom amphiphile with the commercial SDBS and salting with $\mathrm{NaCl}$.

\section{Introduction}

Single-walled carbon nanotubes (SWNTs) constitute a whole set of different materials with different properties depending on the chirality, the (n, $m$ ) value, of the tube at hand. The unit cell of a carbon nanotube determines whether a SWNT is semiconducting or metallic, as well as its diameter and thus surface curvature, pyramidalization and chemical reactivity [1],[2]. As-produced SWNT samples usually consist of a large variety of different nanotubes. Working with as-produced SWNTs means thus working with a wide range of different materials.

In one dimensional systems like SWNTs, the electronic density of states consists of a series of very narrow peaks arranged symmetrically around the Fermi level. As transitions between states of equal distance to the Fermi level are strongly enhanced, photoluminescence maps (PLE) of carbon nanotubes consist of a series of bright spots. The spectral location of these spots, however, depends unequivocally on the tube chirality and thus reveals the presence of a certain (n, m)-value in a sample [3],[4].

A way to solubilize as well as debundle carbon nanotubes and thus restore their luminescence is the use of surfactants

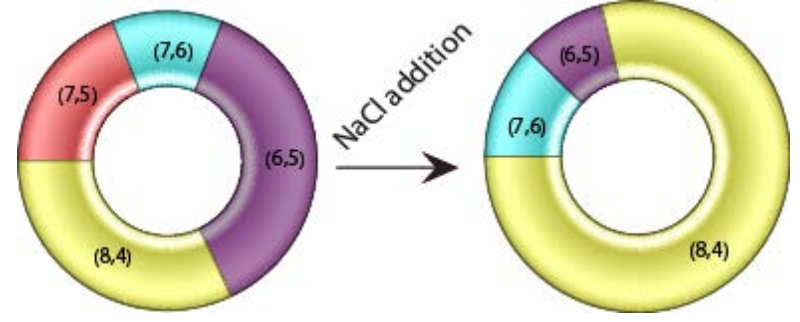

Relative sample composition, before and after the salting out procedure.

like sodium dodecyl sulfate (SDS), sodium dodecylbenzenesulfonate (SDBS), etc. [1],[3],[4].

Recently, much effort has been directed at sorting SWNTs depending on their chirality. Ultradensity gradient centrifugation utilizing a variety of surfactants as well as gradient mediums has been proven to separate nanotubes by type (semiconducting/metallic) as well as by chirality [5]-[7]. Gel affinity chromatography has been shown to yield similar results [5],[8] and the overloading technique has even improved these results [9]. Sadly these methods are very expensive, the yield is low and the methods are very time consuming.

However, we are still far from understanding the interaction between noncovalently attached surfactants and carbon nanotubes. Phase-separation has achieved separation in solution between small- and big-diameter tubes or between metallic and semiconducting tubes [10]. Several efforts [11]-[13] were aimed at selecting nanotubes of one specific chirality by using highly specialized surfactants. In an earlier work we reported on a series of polyglycerol based amphiphiles to achieve efficient SWNT dispersion and individualization in water and showed that by altering the aromaticity of the suspension, we could alter the composition of 

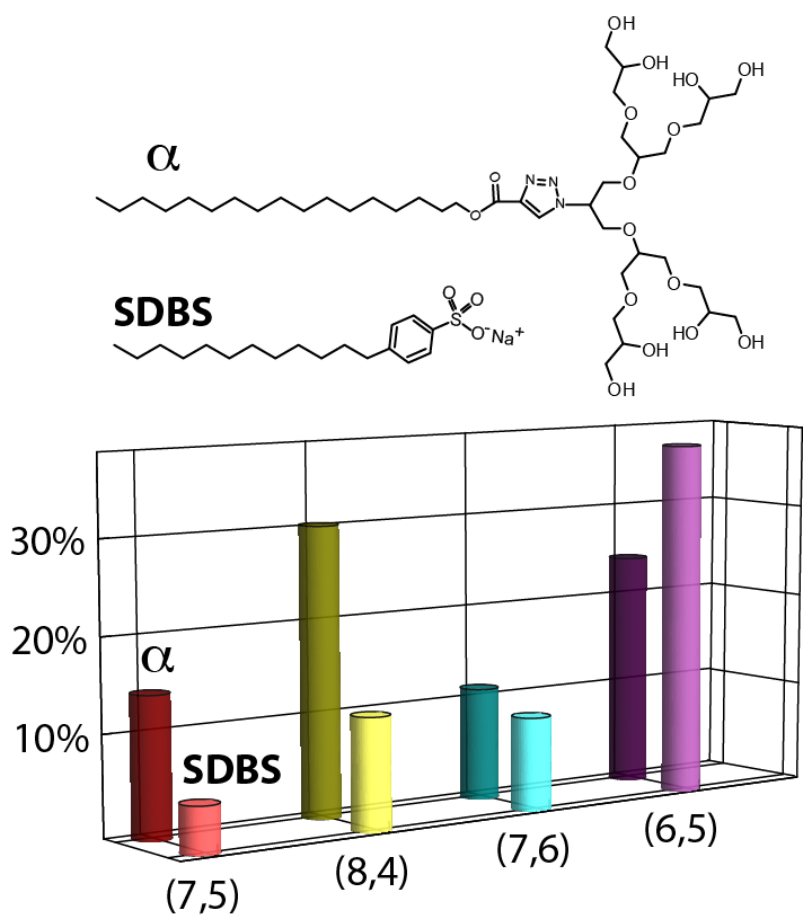

Figure 1: Upper panel: Molecular structure of our custom amphiphile $\boldsymbol{\alpha}$ and of the commercially available SDBS. Lower panel: Comparison between the relative compositions of the nanotube suspensions solubilized with $\boldsymbol{\alpha}$ and SDBS.

the suspensions [14],[15]. The selective interaction between specific polymers and carbon nanotubes has been also shown to lead to chirality enrichement [16],[17]. In this work we study the affinity of a polyglycerol based amphiphile towards nanotubes of a given chirality. We will demonstrate how this might lead to chirally sensitive nanotube selection.

\section{Experimental details}

We will refer to amphiphile used in this work as $\boldsymbol{\alpha}$. For details on its synthesis and characterization please refer to the compound $\mathrm{C}_{2}$ of Ref. [14]. Its molecular structure is depicted in Fig. 1, together with the structure of the commercially available SDBS. $\boldsymbol{\alpha}$ consists of a hydrophilic polyglycerol dendron for water solubility and a hydrophobic alkyl chain to attach onto the nanotube. We found that the linker between the two parts drastically influences the intensity of the interaction of the surfactants with specific laola families of nanotubes $(q=2 n+m=$ fixed) [14]-[20]. The samples were prepared in aqueous solution using an initial nanotube concentration of $0.01 \mathrm{~g} / \mathrm{L}$ and an amphiphile concentration of $10^{-4} \mathrm{Mol} / \mathrm{L}$. After one hour of tip sonication with a power of $60 \mathrm{~W}$, they were centrifuged at $31000 \mathrm{~g}$ and $27^{\circ} \mathrm{C}$ for 60 minutes. The supernatant was used for the experiments.
We used a Bandelin SonoPlus HD 2070 with a maximum power of $200 \mathrm{~W}$. The samples were centrifuged using a Hettig Mikro 220R centrifuge with an 1195-A rotor. We used CoMoCAT nanotubes, produced by SouthWest NanoTechnologies (SWeNT, SG 76) and all belonging to the same production batch. The tubes have a diameter of $(0.9 \pm 0.3)$ nm and a high aspect ratio (1000).

PLE measurements were taken using a Fluorlog-3 Spectrofluometer by Horiba Jobin Yvon. The excitation was provided by a xenon lamp with a range of $240 \mathrm{~nm}-1000 \mathrm{~nm}$, the signals were recorded using a nitrogen cooled InGaAs detector with a range of $800 \mathrm{~nm}-1600 \mathrm{~nm}$.

\section{Results and discussion}

We took the PLE maps with an excitation range of $550 \mathrm{~nm}$ $720 \mathrm{~nm}$ and an emission range of $900 \mathrm{~nm}-1400 \mathrm{~nm}$. The intensity of each spot in the PLE map provides information about the relative abundance of the different chiralities. The lower panel of Fig. 1 reports the composition of dispersions using, respectively, $\alpha$ and SDBS. Their different solubilizing ability makes the $(8,4)$ species the most abundant with $\boldsymbol{\alpha}$ and the $(6,5)$ with SDBS. The PLE map of the $\boldsymbol{\alpha}$-coated SWNTs sample shows a large variety of different nanotubes (Fig. 2a). As some of the tubes exhibit only very weak PLE signal, like the $(11,1)$ tube, they were excluded from the following analysis.

Also the spectral position of the tubes' bands highly depends on environmental effects like the surfactant used in nanotube suspension [21],[22]. This can be exploited to monitor the surfactant substitution: When the surfactant is exchanged, the peaks move from one position to the other [20]. Accordingly, starting from samples solubilized using our amphiphiles $\boldsymbol{\alpha}$, we stepwise added SDBS to our suspensions and monitored the PL intensities at the initial positions of the maxima (highlighted by the white lines in Fig. 2b-e). As a result of the SDBS addition, we observed a blue shift of up to $40 \mathrm{~nm}$ in the emission and $10 \mathrm{~nm}$ in the excitation. This blue shift is shown in Fig. 2b-e for the $(8,4)$ tube for SDBS concentrations of $0 \mathrm{mMol} / \mathrm{L}, \quad 0.38 \mathrm{mMol} / \mathrm{L}$, $0.63 \mathrm{mMol} / \mathrm{L}$ and $0.88 \mathrm{mMol} / \mathrm{L}$ respectively. The peak position does not change substantially between SDBS concentrations of $0 \mathrm{mMol} / \mathrm{L}$ and $0.38 \mathrm{mMol} / \mathrm{L}$ (Fig. $2 \mathrm{~b}$ and $\mathrm{c}$ ). However, beyond a certain SDBS concentration, the surfactant starts replacing $\boldsymbol{\alpha}$ and we observe a shift in the peak positions towards its value for a sample using only SDBS as a surfactant (Fig. 2c,d). We attribute this to the replacement of $\boldsymbol{\alpha}$ with SDBS. The resulting data (intensity vs. SDBS concentration) were fitted using the Hill equation [11],[23].

$$
I=\frac{\left[C_{S D B S}\right]^{\chi}}{\left[K_{a}\right]^{\chi}+\left[C_{S D B S}\right]^{\chi}}
$$




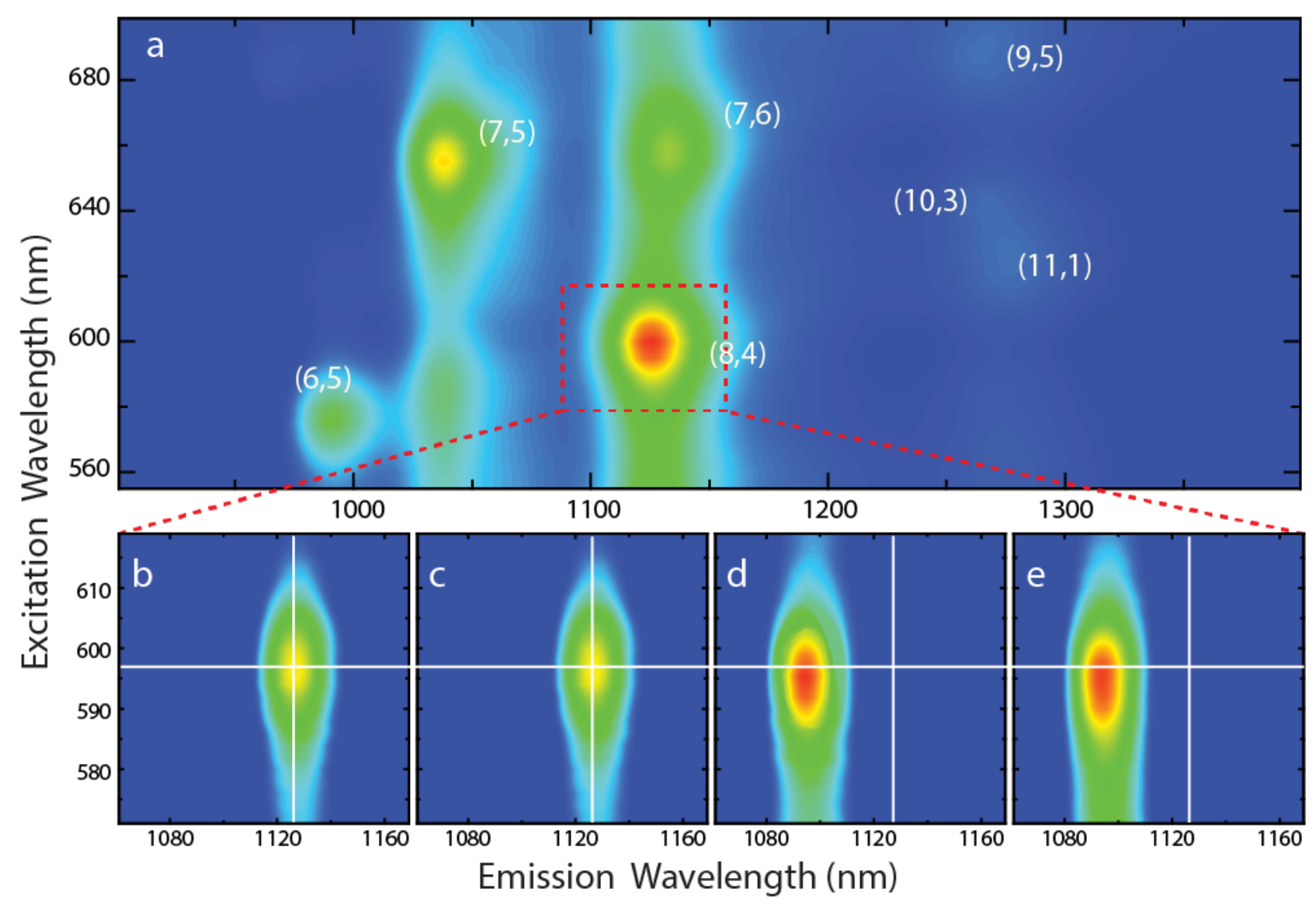

Figure 2: When adding SDBS to a $\boldsymbol{\alpha}$-coated SWNTs dispersion, the luminescence bands shift towards higher energies. The SDBS concentrations are: $0 \mathrm{mMol} / \mathrm{L}$ (b), $0.38 \mathrm{mMol} / \mathrm{L}$ (c), $0.63 \mathrm{mMol} / \mathrm{L}$ (d), and $0.88 \mathrm{mMol} / \mathrm{L}$ (e).

where $I$ is the PLE intensity, $K_{a}$ the relative affinity of $\boldsymbol{\alpha}$ coated SWNTs towards SDBS, CSDBS denotes the SDBS concentration and $\chi$ is the Hill coefficient. In biochemistry the Hill equation is used to describe the binding of a ligand to a macromolecule [11],[23]. We can use it to describe the degree of functionalization of carbon nanotubes with our amphiphile. When successively adding SDBS to the sample, the SDBS will gradualy replace the present surfactant. This change is reflected in the PLE signal. However, the SDBS concentration needed to replace an amphiphile varies from tube to tube and depends on the strength of the interaction between tube and amphiphile. Thus, using the Hills equation it is possible to investigate the adsorption affinity of an amphiphile towards nanotubes of certain chirality.

The exact transition point, the minimum SDBS concentration that favors the replacement of $\boldsymbol{\alpha}$, can be derived from the intensity change at the peak positions in the initial sample without SDBS. Figure 3 shows the results for the $(6,5),(7,5),(7,6)$ and $(8,4)$ tube. The solid lines represent the fitted data using the Hill equation. The value of half occupation, between the regimes where a tube is predominantly functionalized by either one of the two surfactants, is marked with dotted lines.
That the critical concentration varies from tube to tube. The tube-specific values of the SDBS concentrations needed to fully replace $\alpha$ are summarized in Table 1 . For very low SDBS concentrations, all the tubes are coated with $\boldsymbol{\alpha}$. By increasing the SDBS concentration, we will first reach the concentration $\mathrm{C}_{(7,6)}$ at which $(7,6)$ starts getting coated with SDBS and the other tubes remains coated with $\alpha$. Increasing the SDBS concentration to $C_{(7,5)}$, also the $(7,5)$ chiral species will get coated with SDBS and so on by keeping increasing the concentration to $\mathrm{C}_{(6,5)}$ and $\mathrm{C}_{(8,4)}$. By choosing a working point close to $\mathrm{C}_{(8,4)}$, the majority of the tubes are coated with SDBS while only the $(8,4)$ is partly coated with $\alpha$.

Table 1: $K_{a}$ values from the Hill fits.

\begin{tabular}{lllll}
\hline Tube & $(7,5)$ & $(7,6)$ & $(6,5)$ & $(8,4)$ \\
$K_{a}$ & 0.40 & 0.37 & 0.45 & 0.52 \\
\hline
\end{tabular}




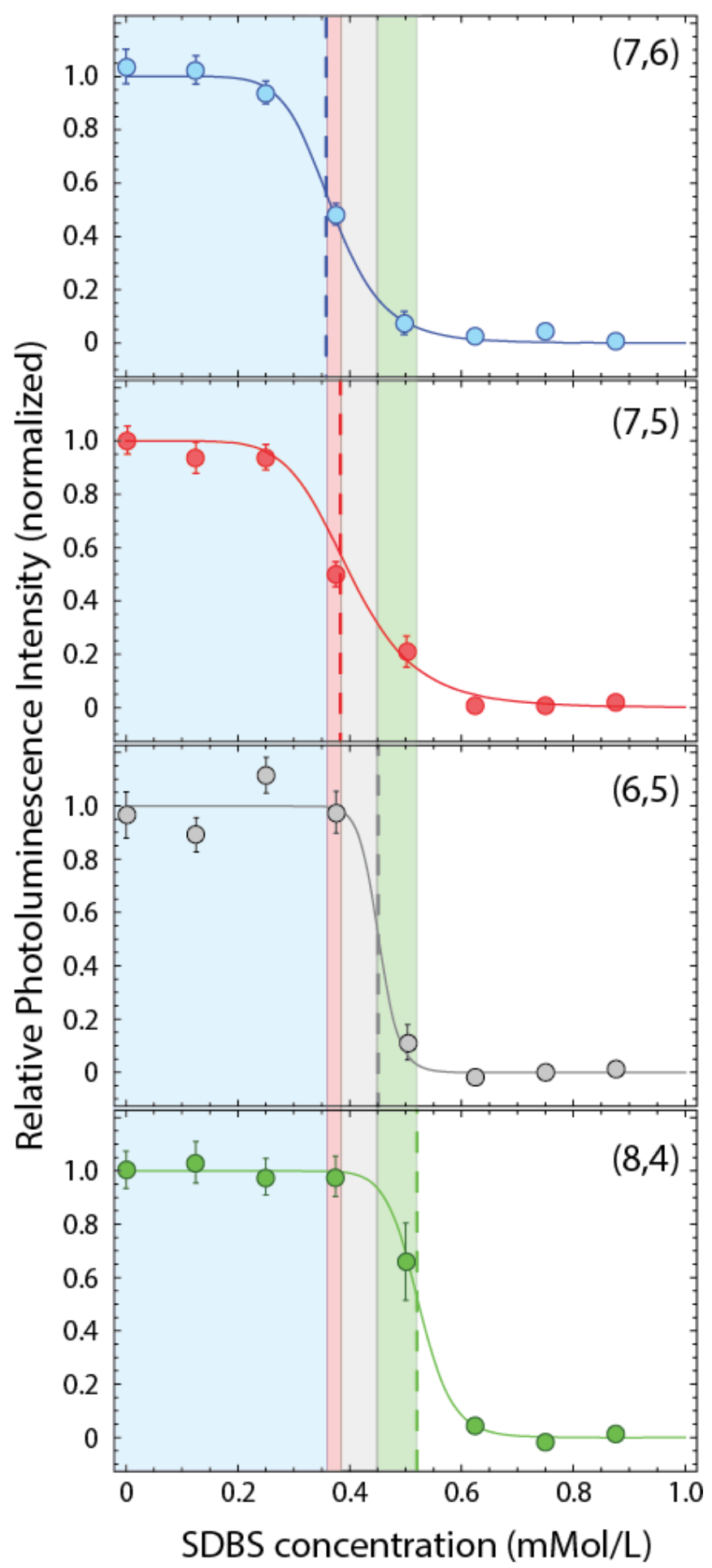

Figure 3: Change of the relative photoluminescence intensity upon increasing SDBS concentration evaluated at the spectral starting position, without any SDBS addition ( $\mathrm{C}_{\mathrm{SDBS}}=0$ ). The sudden intensity drop is a consequence of the surfactant exchange and the resulting shift in peak position. The critical concentration at which the tube is covered half/half with $\alpha$ and SDBS changes from tube to tube and is highlighted with the dashed line.
The fact that each tube exhibit its distinctive value of the critical SDBS concentrations for the surfactant replacement enables exerting control over the composition of the coating of the tubes. We exploit the fact that our customsurfactant $\alpha$ is non-ionic while SDBS is ionic. It is known that ionic surfactants start desorbing from the SWNTs sidewall and trigger their re-aggregation and precipitation when dissolving salts within the solution [24]. Moreover, the presence of charge screening effects reduces the intertube repulsion, further promoting the tube re-aggregation process. We can take advantage of this triggered precipitation, by controlling the coating of targeted species with the non-ionic surfactant while coating with the ionic surfactant the chiral species that we want to get rid of.

Following the approach used by Ju et al. [11], we thus dissolved salt in a solution prepared at $\mathrm{C}_{\text {SDBS }}=\mathrm{C}_{(8,4)}$. After the addition of salt $(\mathrm{NaCl})$ to the solution, the SDBS wrapped are expected to precipitate while the $(8,4)$ tube covered with $\alpha$ (which is an nonionic surfactant) is expected to remain suspended. The choice of the parameters is extremely delicate. For example, even working at the proper surfactant concentration, an excessive amount of salt would induce precipitation of all nanotube species present in solution (Fig. 4a,b). The proper salt concentration was achieved by adding $0.5 \mathrm{Mol} \mathrm{NaCl}$ to the suspension. The sample was then shaken until the salt was dissolved and then recentrifuged.
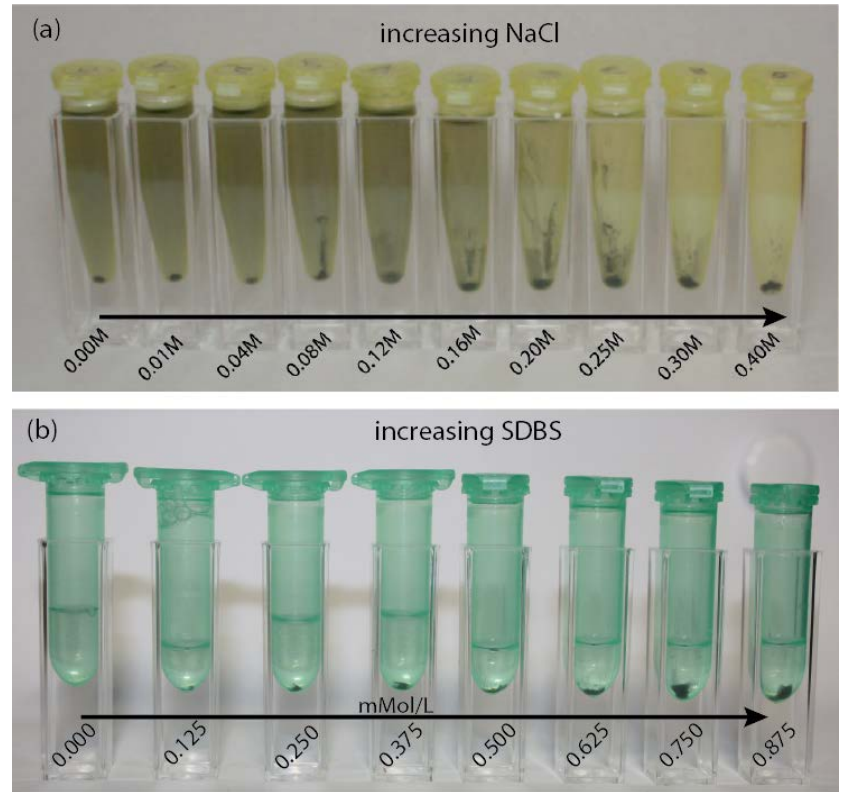

Figure 4: (a) At a fixed SDBS concentration, increasing amount of SDBS triggers precipitation of SWNTs. (b) Stepwise addition of SDBS to the $\boldsymbol{\alpha}$-coated tubes and salting triggers the precipitation of target species. 

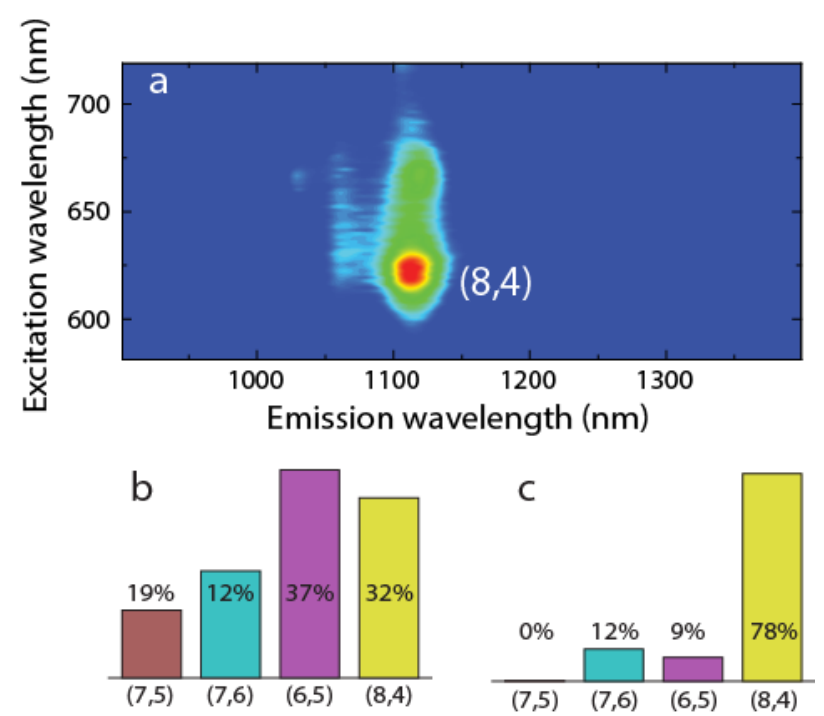

Figure 5: (a) PLE map after salting the $\boldsymbol{\alpha} / \mathrm{SDBS} / \mathrm{CoMoCAT}$ sample. At an SDBS concentration of $0.5 \mathrm{mMol} / \mathrm{L} \mathrm{NaCl}$ was added to the sample. It was then shaken and centrifuged. It is evi- dent that this PLE map reveals an absence of most of the nanotube peaks. The $(8,4)$ tube, however, is clearly visible. (b,c) Relative intensities before (b) and after (c) the salting out procedure. The picture shows the PLE intensities of the $(7,5),(7,6),(6,5)$ and $(8,4)$ tube. After the salting out procedure, we find a significant enrichment of the $(8,4)$ tube.

Under these conditions, the $(8,4)$ tube (which is covered half by $\boldsymbol{\alpha}$ and half by SDBS) still remains in suspension while the other species (covered only by SDBS) precipitate. The resulting PLE map (Fig. 5) shows a strong enrichment of the $(8,4)$ tube to about $80 \%$ of the sample (Fig. 4 b,c). Only a small fraction (ca. 20\%) of the $(8,4)$ tubes was lost in the process. This opens up new possibilites for nanotube sorting. Recently we have shown that the chiral selectivity of similar amphiphiles can lead to large differences in the sample composition [15], [18]. We used polyglycerol amphiphiles consisting of different aromatic groups between head and tail. PLE maps of carbon nanotubes solubilized using those amphiphiles revealed strong differences in the relative luminescence intensity and thus chiral selectivity.

\section{Conclusions}

Combining the selectivity of specialized amphiphiles towards selected chiralities with their relative stability upon environmental changes, we can direct tube separation in a controlled way. This work serves as a proof of principle that could be easily extended to other classes of surfactants responding to different kind of stimuli (Temperature, PH, irradiation and so on), where the only ingredient required is the responsivity of only one of the surfactants to the external stimulus. As the surfactants interaction with the tubes can be tuned by changing the morphology of the linker and the intermediate core, selective precipitation could provide fast large-scale chiral enrichment of different targeted carbon nanotube suspensions by simple, unexpensive technological means.

Acknowledgements The authors would like to thank Britta Trappmann and Rainer Haag for the synthesis of the amphiphile $\boldsymbol{\alpha}$. The authors gratefully acknowledge the German Research Foundation (DFG via SFB 658, subproject A6) and the Focus Area NanoScale of the Freie Universität Berlin for financial support.

\section{References}

[1] A. Hirsch and O. Vostrowsky, Top Curr Chem 245, 193237 (2005).

[2] S. Reich, C. Thomsen, and J. Maultzsch, Carbon Nanotubes Basic Concepts and Physical Properties (WileyVCH, Weinheim, 2004).

[3] S. M. Bachilo et al. Science 298, 23612366 (2002).

[4] M. J. O’Connell, S. M. Bachilo, C. B. Huffman, V. C. Moore, M. S. Strano, E. H. Haroz, K. L. Rialon, P. J. Boul, W. H. Noon, C. Kittrell, J. P. Ma, R. H. Hauge, R. B. Weisman, and R. E. Smalley, Science, 297, 593596 (2002).

[5] K. Moshammer, F. Hennrich, and M. M. Kappes, NanoRes 2, 599-606 (2009).

[6] M. S. Arnold, A. A. Green, J. F. Hulvat, S. I. Stupp, and M. C. Hersam, Nature Nanotechnology 1, 60-65 (2006).

[7] Y. Maeda et al., J. Am. Chem. Soc. 127, 10287-10290 (2005)

[8] T. Tanaka, Y. Urabe, D. Nishide, and H. Kataura, Applied Physics Express 2, 125002 (2009).

[9] H. Liu, D. Nishide, T. Tanaka, and H. Kataura, Nature Communications 2, 309 (2011).

[10] C. Y. Khripin, J. A. Fagan, and M. Zheng, J. Am. Chem. Soc. 135, 6822 (2013).

[11] S. Ju, J. Doll, I. Sharma and F. Papadimitrakopolos, Nature Nanotechnology 3, 356-362 (2008).

[12] S. Kim, Z. Kuang, J. Grote, B. L. Farmer, and R. Naik, Nano Lett. 8, 4414 (2008).

[13] R. Marquis et al., Nano Lett. 8, 1830 (2008).

[14] A. Setaro et al., ChemPhysLett 493, 147-150 (2010).

[15] A. Setaro et al., Phys. Status Solidi B 252, 2536 (2015).

[16] F.K. Brunecker, F. Schöppler, and T. Hertel, J. Phys. Chem. C 120, 10094 (2016).

[17] Y. Kato, A. Inoue, Y. Niidome, and N. Nakashima, Scientific Reports 2, 733 (2012).

[18] A. Setaro et al., Phys. Status Solidi B 247, 2758 (2010).

[19] C. Popeney et al., Chem. Phys. Chem., 13, 203(2012).

[20]P. Bluemmel et al., Phys. Status Solidi B 248, 2532 (2011).

[21] J. H. Choi, M. S. Strano, Appl. Phys. Lett. 90, 223114 (2007).

[22] T. Ando, Physica E: Low-dimensional Systems and Nanostructures, 43, 798-803 (2010).

[23] J. M. Berg, J. L. Tymoczko, and L. Stryer, Biochemistry, 5th Edition (W H Freeman, 2002).

[24] S. Niyogi et al., J. Am. Chem. Soc. 129, 1898 (2007). 